\title{
The E2FI/DNMTI Axis Is Associated With the Development of AR Negative Castration Resistant Prostate Cancer
}

\author{
Conrad David Valdez, ${ }^{1,2}$ Lakshmi Kunju, ${ }^{3,4}$ Stephanie Daignault, ${ }^{4}$ \\ Kirk J. Wojno, ${ }^{5}$ and Mark L. Day ${ }^{1,2,4 *}$ \\ 'Department of Urology, University of Michigan, Ann Arbor, Michigan \\ ${ }^{2}$ Program in Cellular and Molecular Biology, University of Michigan, Ann Arbor, Michigan \\ ${ }^{3}$ Department of Pathology, University of Michigan, Ann Arbor, Michigan \\ ${ }^{4}$ University of Michigan, Ann Arbor, Michigan \\ ${ }^{5}$ Comprehensive Urology Division of Michigan Healthcare Professionals, University of Michigan, \\ Ann Arbor, Michigan
}

\begin{abstract}
BACKGROUND. Research on castration resistant prostate cancer (CRPC) has focused primarily on functional alterations of the androgen receptor (AR). However, little is known about the loss of AR gene expression itself and the possible contribution of AR negative cells to CRPC.

METHODS. Human and murine prostate cancer tissue microarrays (TMAs) were evaluated with antibodies specific for E2F1, DNA methyltransferase 1 or AR. The human prostate cancer TMA consisted of clinical samples ranging from normal tissue to samples of metastatic disease. The murine TMA was comprised of benign, localized or metastatic prostate cancer acquired from TRAMP mice treated with castration and/or 5'-Aza-2'-deoxycytidine (5Aza).

RESULTS. Immunohistochemical analysis revealed increased nuclear DNMT1 staining in localized PCa $(P<0.0001)$ and metastatic PCa $(P<0.0001)$ compared to normal tissue. Examination of specific diagnoses revealed that Gleason seven tumors exhibited greater nuclear DNMT1 staining than Gleason six tumors $(P<0.05)$ and that metastatic tissue exhibited greater levels of nuclear DNMT1 than Gleason seven tumors $(P<0.01)$. Evaluation of the murine tissue cores revealed that $8.2 \%$ and $8.1 \%$ of benign tissue cores stained positive for E2F1 and DNMT1 respectively, while $97.0 \%$ were AR positive. Conversely, $81 \%$ and $100 \%$ of tumors were positive for E2F1and DNMT1 respectively. This was in stark contrast to only $18 \%$ of tumors positive for AR. Treatment of mice with 5Aza reduced DNMT1 staining by $30 \%$, while AR increased by $27 \%$.

CONCLUSIONS. These findings demonstrate that the E2F1/DNMT1 inhibitory axis of AR transcription is activated during the emergence of CRPC. Prostate 73:1776-1785, 2013.
\end{abstract}

(C) 2013 Wiley Periodicals, Inc.

KEY WORDS: methylation; DNMT1; E2F1; androgen receptor; tissue microarray; immunohistochemistry

\section{INTRODUCTION}

Castration resistant prostate cancer (CRPC) continues to be the leading cause of prostate cancer related deaths. Research on this disease has focused primarily on the functional deregulation of androgen receptor (AR) during progression to CRPC, however, little is known about the loss of AR gene expression itself and the possible contribution of AR negative cells to CRPC. A significant decrease in AR expression coincident with the onset of CRPC has been demonstrated in
Grant sponsor: National Institutes of Health; Grant numbers: R01 DK-056137; R01 DK-066610; Grant sponsor: Department of Defense; Grant number: W81XWH-08-1-0035.

Disclosure/Conflict of Interest: There are no author disclosures or conflicts of interest, recent, present or anticipated.

${ }^{*}$ Correspondence to: Mark L. Day, Comprehensive Cancer Center, University of Michigan, 1500 East Medical Center Drive, Ann Arbor, MI 48109-5942. E-mail: mday@umich.edu

Received 27 February 2013; Accepted 6 July 2013

DOI 10.1002/pros. 22715

Published online 4 September 2013 in Wiley Online Library (wileyonlinelibrary.com). 
prostate tumors recurring after anti androgen therapy [1]. Immunohistochemical (IHC) analysis has also revealed a significant decrease in AR expression in prostate tumors from patients who failed combination hormone therapy compared to untreated individuals [2]. More recently, we reported that metastatic lesions from CRPC patients revealed a significant reduction in AR staining when compared to hormone naïve cases [3]. Taken together, these findings suggest that reduced $\mathrm{AR}$ expression may contribute to the development of CPRC; however, complete elucidation of these inhibitory mechanisms has not been fully described.

E2F1 is a transcription factor that plays a role in driving S-phase transit of the cell cycle during cellular proliferation [4]. A genome wide microarray study evaluating gene regulatory signatures associated with cancer verified that more than half of the signatures analyzed were E2F1 target genes [5]. Using a metastatic prostate cancer tissue microarray (TMA), we found significant increases in E2F1 staining in metastatic CRPC samples compared to hormone naïve cases that were significantly associated with concurrent reduction of AR staining in the same samples [3]. Another study reported that E2F1 cDNA was elevated in advanced prostate cancer and lymph node metastases and also demonstrated that the majority of the tumor cell nuclei in tumors associated with biochemical recurrence exhibited strong E2F1 staining [6]. We had previously reported that the activation of the DNMT1 gene is specifically mediated by E2F1 in several prostate cancer models and that catalytic inhibition of DNMT1 prevents prostate tumor formation and metastatic progression [7]. Based on these studies, several mechanisms by which elevated E2F1 drives prostate cancer progression have been postulated including a prolonged hyper-proliferative state of tumor cells or the epigenetic silencing of tumor suppressor genes through aberrant activation of DNMT1.

DNMT1 is a member of the DNA methyltransferase family that is responsible for maintaining methylation patterns located in CG dinucleotide-rich regions within genomic DNA. Methylation of DNA contributes to genetic silencing by cooperating with other epigenetic mechanisms to either block transcriptional complexes or induce conformational changes in the DNA structure that inhibit transcription [8]. Aberrant DNMT1 expression and function are associated with the progression of various human tumors and elevated DNMT1 levels have been reported in the metastatic progression of many human cancers $[9,10]$. DNMT1 protein has also been reported to be elevated in prostate tumors compared to benign prostate tissue and that DNMT1 mRNA expression is increased in advanced prostate cancer and in experimental cell- based models that recapitulate CRPC [11,12]. A genome wide cDNA microarray study conducted by Tomlins et al. [13] was the first to reveal elevated DNMT1 cDNA in CRPC compared to primary prostate cancer; however, little more is known about the relationship between DNMT1 and the development of CRPC in patients. While it does not fully recapitulate the histo-pathological progression of human prostate cancer, the murine transgenic adenocarcinoma of the mouse prostate (TRAMP) model has been very useful in studies examining increased DNMT1 expression during the progression to poorly differentiated and metastatic disease $[13,14]$. Using this model, we have shown that the catalytic inhibition of DNMT with 5Aza treatment prevented both the histo-pathological progression and associated lethality in TRAMP mice [7]. Extensive efforts to reveal molecular markers in vastly heterogeneous prostate cancer cases have identified a number of consistently hyper-methylated genes that may represent the catalytic signature of DNMT1 in prostate cancer progression [15]. Collectively, these observations support a functional role for DNMT1 during prostate cancer progression that may include, directly or indirectly, the repression of $A R$ gene transcription in the development of CRPC.

We have recently described a novel mechanism by which AR transcription is repressed through E2F1mediated induction of the DNA methyltransferase 1 (DNMT1) gene [16]. Based on this evidence, we postulated that this mechanism may play a role in the repression of AR in a subset of CRPC patients. To help establish the existence of this regulatory axis in CRPC, we examined the expression of E2F1, DNMT1, and AR in samples of human CRPC and in murine models of CRPC

\section{MATERIALS AND METHODS \\ Human Prostate Cancer Progression Tissue Microarray Components}

The tissue donation and rapid autopsy programs was approved by the Institutional Review Board of the University of Michigan. One hundred ninety cores of pathologically confirmed prostate tissue from 90 patients were used to construct the TMA used for this analysis. The cores contained normal, benign prostatic hyperplasia (BPH), BPH stromal nodules, prostate intraepithelial neoplasia (PIN), prostatic atrophy, proliferative inflammatory atrophy (PIA), and both localized and metastatic prostate cancer tissues. The component of interest was scored on each fragment containing heterogeneous tissue. Benign prostate tissue was acquired by cystoprostatectomy and transurethral resection for benign from 30 patients. Precancerous (PIN and hyperplasia) and localized disease 
samples were obtained from radical prostatectomy specimens from patients who had their prostate removed for prostate cancer. Metastatic samples from distant tissue sites were acquired through rapid autopsy from 10 patients who failed androgen deprivation therapy. The breakdown for all tissue in the human prostate cancer progression TMA was; normal $\mathrm{n}=27$ cores, localized cancer $n=59$ cores, metastatic $n=19$ cores, G6 $\mathrm{n}=8$ cores, and G7 $\mathrm{n}=27$ cores.

\section{TRAMP Tissue Microarray Components}

We utilized tissue from the TRAMP model [17] to create a 333 core TMA consisting of tissue from 111 wild-type strain (C57BL/6) matched control animals and TRAMP mice that were either castrated, sham operated, or treated with $5^{\prime}$-Aza-2'-deoxycytidine (5Aza). 5Aza treatment and surgical orchiectomy were performed independently or in combination as described [18]. We considered the majority of TRAMP mice castrated at 15 weeks of age to recapitulate CRPC, based on evidence showing greater than $80 \%$ of regressed TRAMP tumors following castration return after 24 weeks [19]. All tissues evaluated in the present study, were taken from mice sacrificed at 24 weeks of age. The TRAMP TMA was assessed independently for the expression of DNMT1, E2F1, or AR and the immuno-positive percentage of cores evaluated with each antibody.

\section{Analysis of E2FI, AR, and DNMTI Protein Expression Via Immunohistochemistry}

Both the human prostate cancer TMA and the TRAMP TMA were evaluated using standard biotinavidin complex immunohistochemistry and the following antibodies; DNMT1 (ab19905, Abcam), E2F1 (KH95, Calbiochem) and AR (N-20, Santa Cruz). Two board certified GU pathologists examined all tissue cores and scored nuclear DNMT1 immunostaining on a scale from 0 to 3 (0-none, 1-weak, 2-moderate, and 3-intense). A mean nuclear intensity (MNI) score was generated for each pathologically characterized group from both TMAs. The MNI was calculated for each cohort by dividing the sum of the nuclear intensity values (0-3) by the total number of tissue cores $(\mathrm{MNI}=$ staining intensity score sum/total number of tissue cores). Statistical significance between MNI values were obtained using Tukey's multiple comparison test.

\section{RESULTS}

\section{Analysis of DNMTI Expression in Human Tissues}

We have previously reported that the increased expression of E2F1 protein correlates with reduced AR protein levels during prostate cancer progression to CRPC in human samples [3]. Further studies by our group established the existence of an AR repressive mechanism controlled by E2F1-dependent activation of the DNMT1 gene that promotes prostate tumor progression to CRPC in mice $[16,18]$. To begin to establish a patho-physiologic role for DNMT1 in human prostate cancer and the emergence of CRPC, we evaluated a human TMA comprised of benign prostate tissue as well as localized and metastatic prostate cancer tissue. The MNI scores for DNMT1 staining were determined in 27 cores from normal prostate tissue, 59 cores with localized prostate cancer, and 19 metastatic, CRPC cores from distal tissue sites. DNMT1 nuclear staining significantly increased from normal to metastatic prostate cancer (Fig. 1A). The DNMT1 MNI score of 1.95 was significantly $(P<0.0001)$ greater in metastatic cores compared to localized PCa $(\mathrm{MNI}=1.05)$. Normal tissue cores exhibited the least nuclear staining $(\mathrm{MNI}=0.19)$, which was significantly $(P<0.0001)$ lower than levels observed in either localized or metastatic PCa samples $(P<0.0001$; Fig. 1B).

Further evaluation of this TMA and review of the associated clinical data revealed significant increase in MNI scores corresponding to rising Gleason scores. Tissues with a Gleason score of 7 (G7) showed significantly $(P<0.05)$ greater nuclear DNMT1 staining $(\mathrm{MNI}=1.26)$ than $\mathrm{G} 6$ graded cores $(\mathrm{MNI}=0.5)$ (Fig. 2A and B). Metastatic cancer samples (MNI $=1.95)$ with the highest nuclear DNMT1 levels were significantly $(P<0.01)$ higher than G7 staged localized prostate cancer (Fig. 2B). These data demonstrated that localized prostate cancer exhibits an increased DNMT1 MNI score that is associated with increasing Gleason score. Additionally, nuclear DNMT1 staining was strongest in metastatic tissues from patients who also failed androgen-deprivation therapy. These data demonstrated a positive association between DNMT1 expression and increasing Gleason stage, metastasis and development of CRPC.

\section{Analysis of AR Regulation Through the E2FI/ DNMTI Axis in theTRAMP Mouse}

We had previously suggested that there existed an inhibitory axis by which E2F1 and DNMT1 could repress the transcription of AR during the progression of prostate tumors in TRAMP mice. To test this hypothesis, we examined prostate tissue from male TRAMP mice, in which the retinoblastoma tumor suppressor $(\mathrm{pRb})$ is inactivated by the prostate-specific expression of SV-40 large T antigen (LgT), resulting in increased E2F1 activity in the prostate gland. Prostate tissue from TRAMP mice with poorly differentiated 
A

Normal Tissue

Localized Cancer

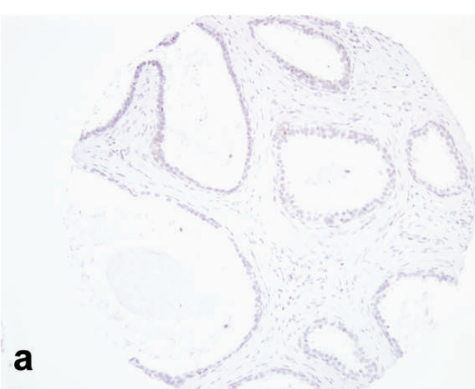

a

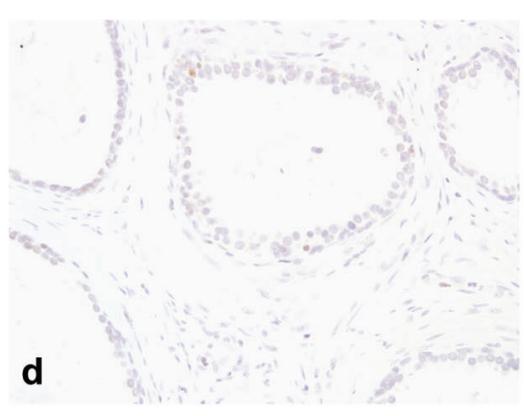

b

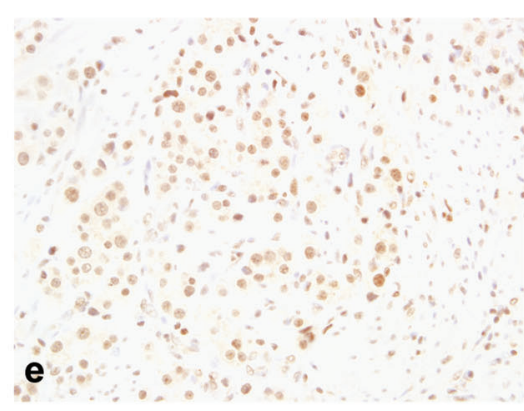

Metastatic Cancer
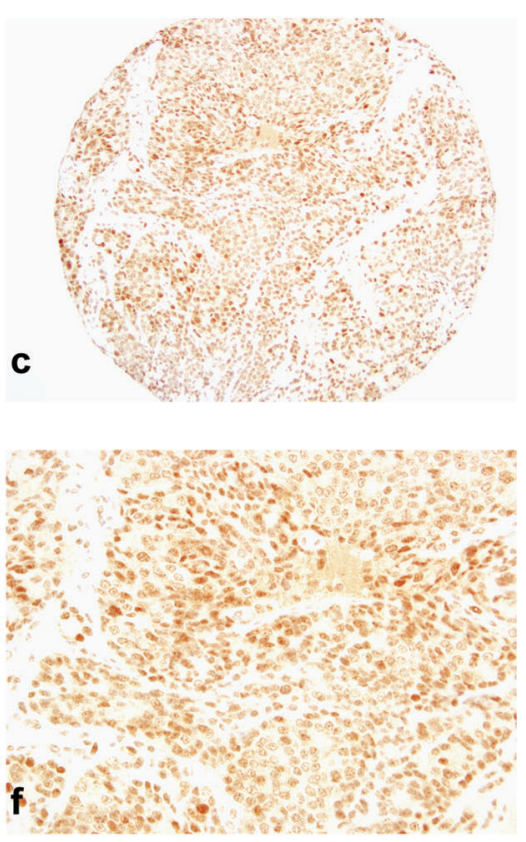

B

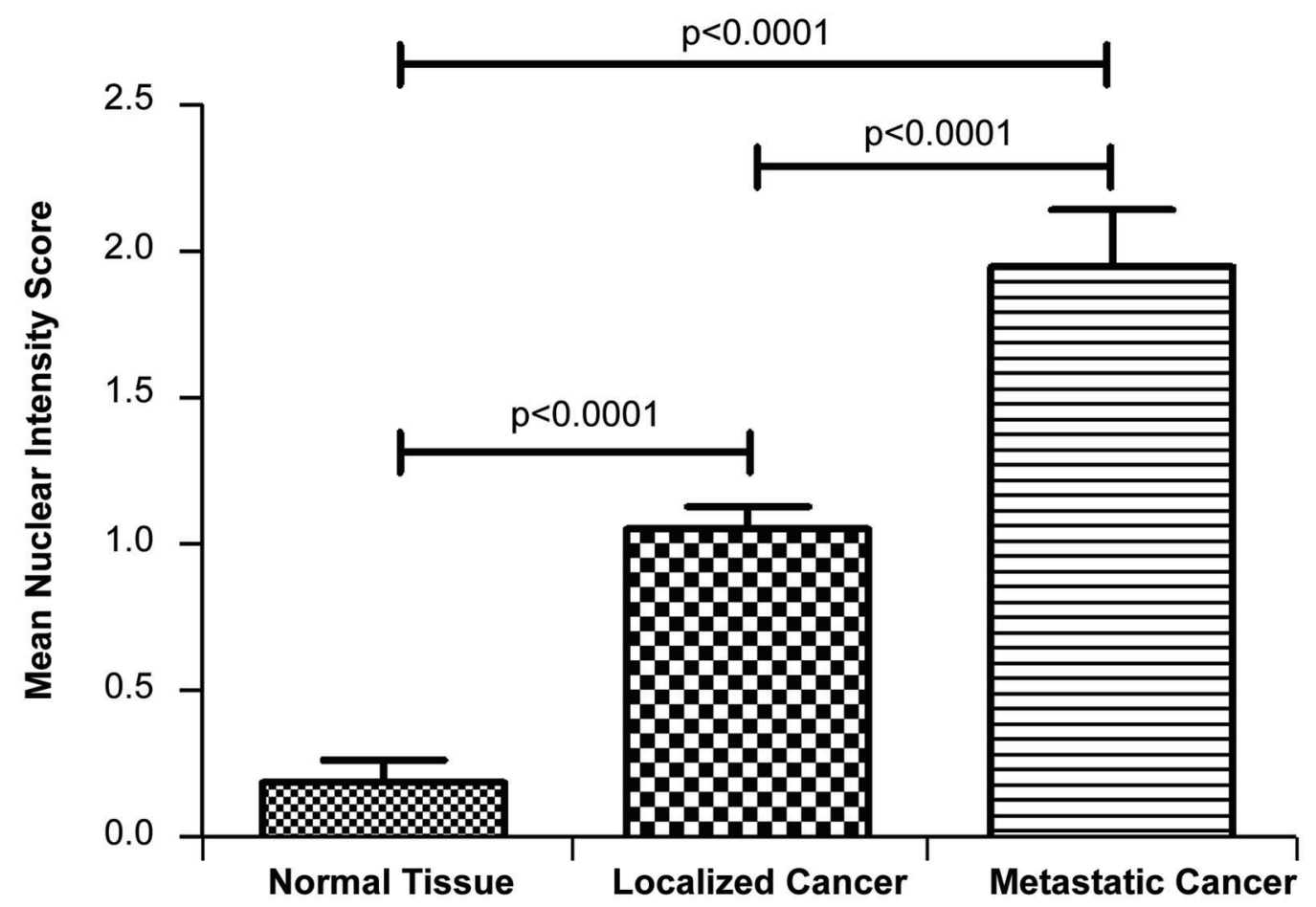

Fig. I. Nuclear DNMTI expression during prostate cancer progression. A: Histological representation from a human prostate cancer progression TMA consisting of normal, localized, and metastatic prostate cancer (magnification; $20 \times(\mathbf{a}-\mathbf{c})$ and $40 \times(\mathbf{d}-\mathbf{f})$ ). B: Mean nuclear intensity scores are presented in a bar graph for each diagnosis (normal $n=27$ cores, localized cancer $n=59$ cores, and metastatic $\mathrm{n}=19$ cores). Tukey's multiple comparison test demonstrates statistical significance $(P$-value $<0.000 \mathrm{I})$. 

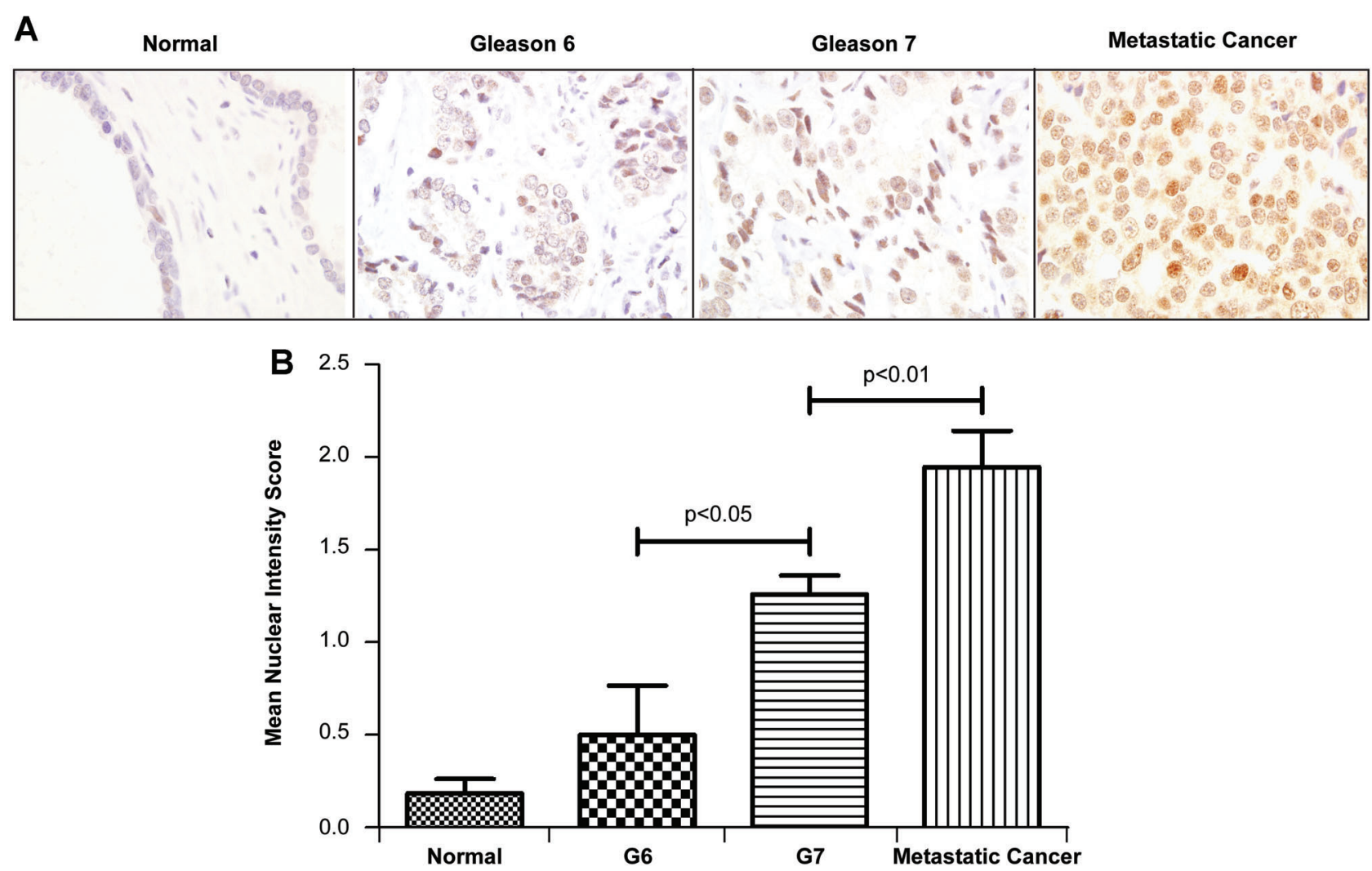

Fig. 2. Nuclear DNMTI expression in Gleason 6 and Gleason 7 prostate tumors. A: Nuclear DNMTI staining shown in normal ( $\mathrm{n}=27$ cores), Gleason 6 (G6) ( $n=8$ cores), and Gleason 7 (G7) ( $n=27$ cores) prostate cancer, and metastatic prostate cancer ( $n=19$ cores). B: The $\mathrm{MNI}$ is represented in a bar graph. Tukey's multiple comparison test demonstrates statistical significance (G6 vs. G7, $P$-value $<0.05$ and G7 vs. metastatic cancer, $P$-value $<0.01$ ).

prostate cancer (PD PCa) stained positive for SV-40 LgT, which was absent in tissues from C57BL/6 control mice (Fig. 3A). E2F1 immunoreactivity was also higher in PD PCa compared to normal tissues (Fig. 3A). Increased levels of the E2F1 target gene, PCNA, were used as an indication of both increased E2F1 activity and cellular proliferation in the TRAMP tumors. Epithelial cells in control (normal) prostate tissues were negative for PCNA staining (Fig. 3A), indicating both low E2F1 activity and a low proliferative index. In contrast, PCNA protein levels were much higher in poorly differentiated tumor tissue derived from TRAMP mice at 24 weeks. This increase in cell proliferation and E2F1 activity was associated with a concomitant increase in nuclear DNMT1 staining and subsequent decreases in AR protein. Control tissues revealed strong AR nuclear staining. These results demonstrated that increased E2F1 expression correlated with increased cell proliferation and expression of E2F-target genes (PCNA and DNMT1) with a concomitant down-regulation of AR in prostate epithelial cells during prostate cancer progression in the TRAMP model. Only 4/49 (8.2\%) and 5/62 (8.1\%) of the benign tissue cores stained positive for E2F1 and
DNMT1 respectively, while 65/67 (97.0\%) of the cores stained positive for AR. Conversely, a large proportion of the tissues diagnosed with cancer stained positive for E2F1 (59/73 (80.8\%)) and DNMT1 (80/80 (100\%)) compared to $(12 / 67(17.9 \%))$ of the cores that stained positive for AR (Fig. 3B). The observation that high levels of E2F1 and DNMT1 are seen in PD TRAMP tumors that concomitantly express low levels of AR continue to support a regulatory role for the repression of AR transcription by the E2F1/DNMT1 axis in vivo.

\section{Analysis of E2FI, DNMTI, and AR in CRPC of TRAMP Mice}

To determine if a correlation of AR repression with increased levels of E2F1 and DNMT1 existed during the development of CRPC, we compared the immunostaining of these proteins in the mouse TMA. Cores from 5Aza treated and untreated castrated TRAMP mice, which recapitulate CRPC when castrated at 15 weeks of age were examined. As shown in Figure 4, we observed a $30 \%$ decrease in tissues stained with DNMT1 from castrated TRAMP mice receiving 5Aza compared to those treated with PBS. The percent of 

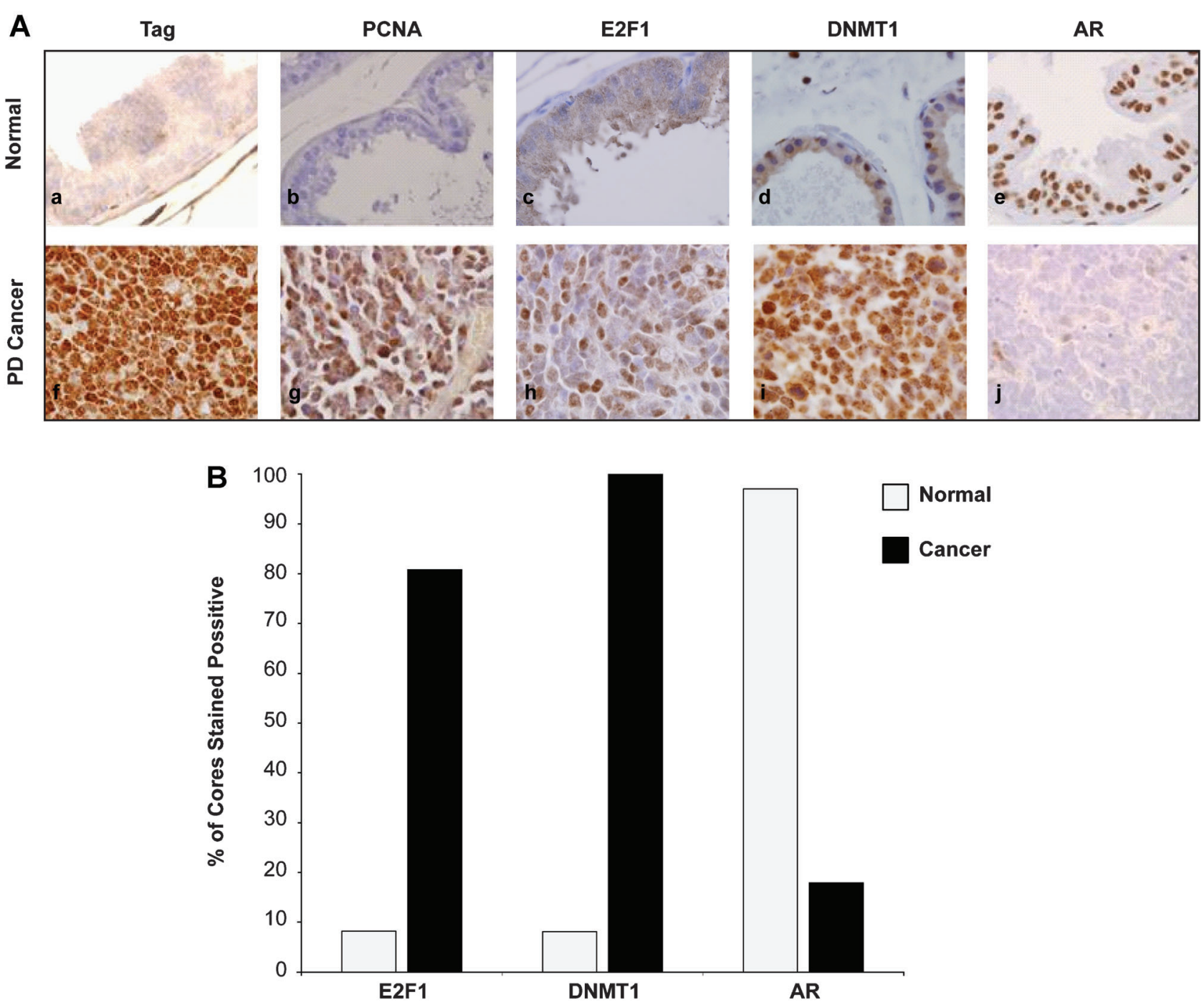

Fig. 3. E2FI, DNMTI, and AR staining TRAMP tissue. A: Immunohistochemical staining of normal prostate tissue from C57BL/6 control mice (panels $\mathbf{a}-\mathbf{e}$ ) at $10 \times$ magnification and prostate cancer from 24 week old TRAMP mice (panels $\mathbf{f}-\mathbf{j}$ ) at $100 \times$ magnification. Sections were stained for LargeTag (panels a and f), PCNA (panels b and g), E2FI (c and h), DNMTI (d and i), and AR (panels e and j). B:The percentage of tissue cores staining positive for either E2FI, DNMTI, or AR are shown as a percent of the total amount of cores evaluated per antibody.

AR positive cores, however, increased by $27 \%$ after 5Aza treatments. No apparent changes were observed in the amount of E2F1 positive tissues taken from untreated versus 5Aza treated mice. Representative tissue from castrated TRAMP mice that maintained normal glandular morphology following 5Aza treatment show intense nuclear AR staining compared to decreases in DNMT1 (Fig. 4A). These data demonstrate that AR levels increase in the absence of DNA methylation and reduced DNMT1 levels in vivo.

\section{DISCUSSION}

The molecular events underlying the clinical recurrence of CRPC remain vague. Based on our previous discovery that AR transcription is inhibited through the E2F1/DNMT1 axis, we set out to establish a mechanism that reveals a functional association with CRPC and also provide the first comprehensive histopathological evaluation of DNMT1 in human prostate cancer. The significant association between prostate cancer progression to CRPC and increased DNMT1 expression supports a plausible utility for DNMT1 as a diagnostic marker for certain prostate cancers. A previous study reports that gastric cancer patients with high epithelial DNMT1 expression have a significantly higher risk of death [20]. Elevated DNMT1 expression was additionally shown to indicate the presence of metastatic prostate cancer in the TRAMP model. Kinney et al. [14] observed a reduction in prostate cancer metastases after DNMT1 levels were knocked down in TRAMP mice. Our study reveals 


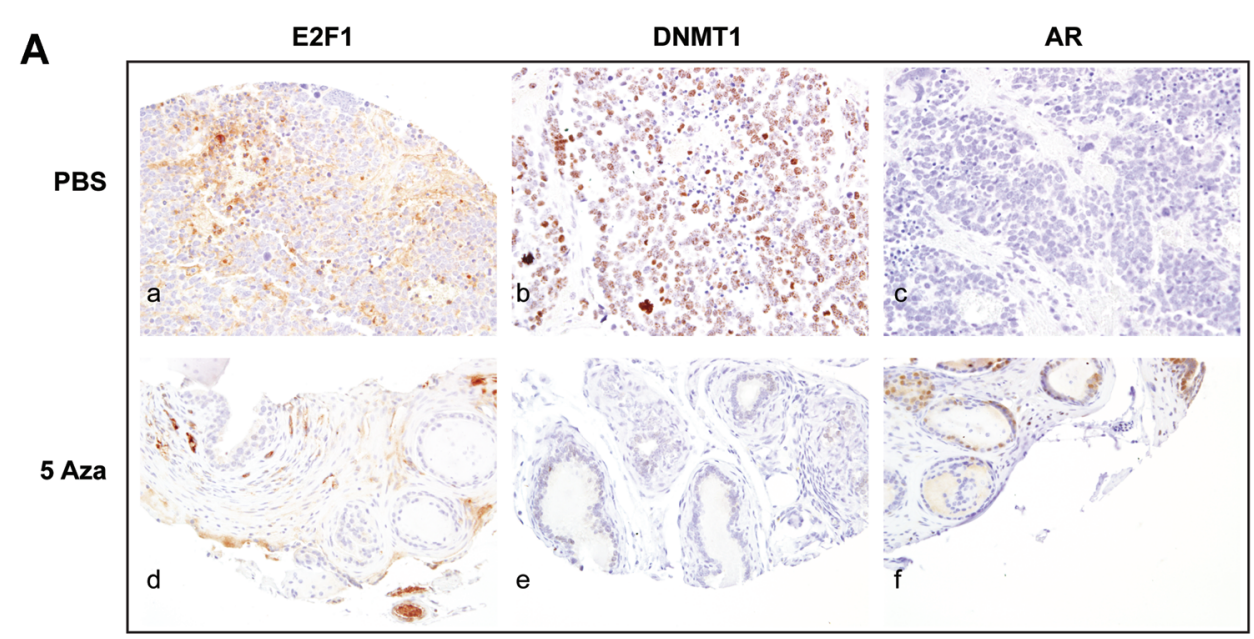

B

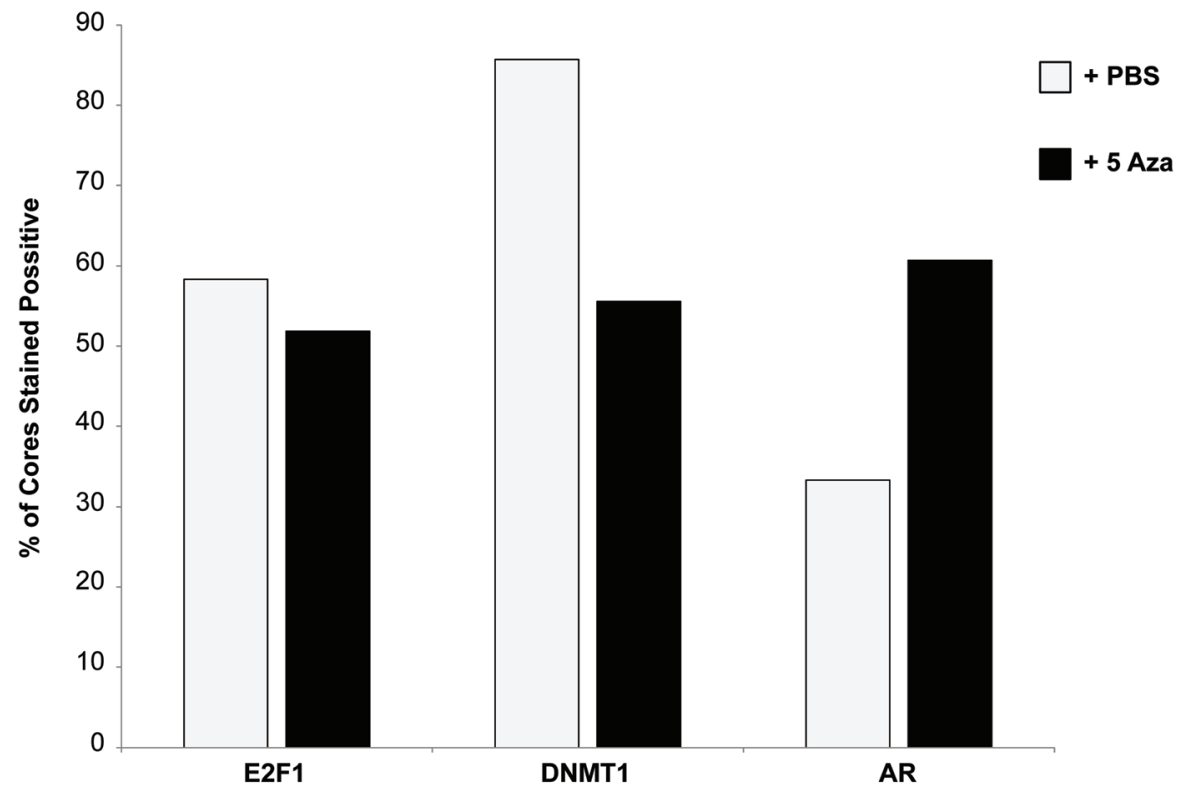

Fig. 4. E2FI, DNMTI, and AR expression in the TRAMP CRPC model. A: E2FI (a,d), DNMTI (b,e), and AR (c,f) immunostained cores represent tissues taken from castrated TRAMP mice treated with either PBS $(a-c)$ or $5^{\prime}-A z a-2^{\prime}$-deoxycytidine $(5 A z a)(d-f)$. B: The percentage of E2FI, DNMTI, and AR positive tissues taken from castrated TRAMP animals are shown as a percent of the total number of tissue cores analyzed for either PBS or $5 \mathrm{Aza}$ treated mice.

DNMT1 expressional changes correlate with stage (localized vs. metastatic) and histological grade, with increased intensity for both more advanced disease and higher grade tumor. The significant difference in MNI between Gleason grade 6 and 7 is of interest for potential clinical application in the setting of active surveillance. There was a relatively even distribution of Gleason $3+4(44 \%)$ and $4+3(56 \%)$ in our sample set, however there were only a small number of $4+4$ and $4+5$ samples for examination and even though these intensity scores were similar to the Gleason 7 group, the small number of $4+4$ and $4+5$ samples lacked sufficient power to draw substantive conclusions. There is an ever expanding repertoire of markers being applied to evaluate small Gleason 6 and 7 grade tumors in an effort to triage patients into active surveillance and definitive treatment categories. Therefore, DNMT1 staining of prostate biopsy samples alone or in combination with other markers may be of clinical value in this setting and deserves further study.

$A R$ repression is one of several molecular events observed in CRPC. We have demonstrated the maintenance of AR levels in experimentally induced CRPC from TRAMP mice after treatments with 5Aza suggesting that AR repression is mediated by DNA methylation and can be prevented by inhibition of DNMT activity. AR is known to be methylated in a 
series of metastatic human prostate cancer cell lines. In addition to being methylated within specific regions, AR gene expression is rescued in androgen independent TSU-PR1 [21] and rat cells [22] after 5Aza treatments. Kinoshita et al. [23] also identified hypermethylated regions within and downstream of the AR promoter that were common amongst AR negative castration resistant cell lines. A study of localized prostate cancer samples revealed that AR gene methylation was specifically occurring during late stage prostate cancer [24]. A comparison of prostate cancer tissues showing AR methylation demonstrated that $100 \%$ of the CpG sites analyzed were methylated in samples from CRPC patients compared to $50 \%$ of the sites being methylated in samples acquired from localized prostate tumors [25]. These data further support a mechanism for AR regulation via methylation in our model of CRPC.

Current studies of AR isoforms have provided supporting evidence of a functional role for specific variants in prostate cancer. AR isoforms contain either C- or N-terminal truncations [26-28]. The AR variants lacking the C-terminal domain are shown to be associated with prostate cancer and disease progression. Expression of the C-terminal truncated AR-V1 and $\mathrm{AR}-\mathrm{V} 7$ variants were observed at increased levels in CRPC tissue samples compared to hormone naïve patient tissue [29]. Guo et al. [30] demonstrated that $\mathrm{AR}-\mathrm{V} 7$ is reacquired for the proliferation of androgen independent 22Rv1 cells grown in the absence of androgens. Less is known about the N-terminal truncated variants AR-A $(87 \mathrm{kDa})$ and AR45 $(45 \mathrm{kDa})$ [26,27]. The isoforms that lack the transactivation domain are shown to disrupt AR activity and reduce cellular proliferation rates [27,31]. Our study utilized an $\mathrm{N}$-terminal directed antibody that fails to recognize the AR-A and AR45 isoforms. However, all C-terminal truncated variants pertinent to a study of CRPC are recognized by the antibody.

DNMT1 facilitates genomic silencing by maintaining the state of DNA methylaion but is also known to utilize de novo pathways. We have previously observed the repression of AR by E2F1 through DNMT1 as an intermediary [16]. Our current study demonstrated that DNMT1 increases during the progression of human prostate cancer to metastatic and CPRC disease. Higher DNMT1 levels may facilitate methylation dependent silencing and suppression of AR activity, thus providing a role for DNMT1 in maintaining abnormal levels of methylation. Robert et al. [32] showed that the down regulation of DNMT1 with anti-sense oligonucleotides in HCT116 colorectal cells significantly increased the amount of unmethylated DNA. High DNMT1 levels and increased genomic methylation have also been reported during the metastatic progression of prostate cancer in the murine TRAMP model [14]. Contrary to these findings, Hoffmann et al. [33] reported that DNMT1 is not responsible for methylation during prostate cancer. However, this study did not globally assess genome-wide methylation. DNMT1 may therefore repress AR expression through methylation during late stage prostate cancer and CRPC.

The substantial increase in $A R$ and decrease in DNMT1 following 5Aza treatment in the TRAMP CRPC model suggests that AR is regulated by DNMT dependent methylation and may represent a therapeutic target. Methylation inhibitors such as $5 \mathrm{Aza}$, were shown to be effective in the treatment of myelodisplastic disease [34], but the short half-life and high toxicity of $5 \mathrm{Aza}$ is currently prohibitive for patient treatment. More suitable treatments may utilize less toxic methylation inhibitors such as Zebularine [35]. Other alternatives might employ the direct targeting of DNMT1 through the use of siRNA oligonucleotides. Knockdown of DNMT1 mRNA was shown to reduce proliferation and increase apoptosis in gastric carcinoma cell lines [36]; however the delivery of siRNA oligonucleotides to tumors remains problematic. Future studies will need to be conducted to fully evaluate the therapeutic potential of DNMT1 as well as the predictive value of DNMT1 in CRPC disease.

\section{CONCLUSIONS}

AR repression through the E2F1/DNMT1 axis may be a mechanism by which cells maintain an undifferentiated yet aberrantly proliferative state during the progression to CRPC. We demonstrated that nuclear DNMT1 like E2F1 increases during the metastatic progression of human prostate cancer and these changes coupled with decreases in AR expression are recapitulated in TRAMP mice with poorly differentiated prostate cancer. Treating castrated TRAMP mice with 5Aza provided us with a better understanding of the molecular changes that occur during the progression to CRPC. From these data we conclude that increases in epithelial AR levels following the inhibition of DNA methylation leads to AR repression that may require DNMT1 catalytic activity during progression to CRPC.

\section{ACKNOWLEDGEMENTS}

This work was supported in part by the National Institutes of Health (R01 DK-056137 and R01 DK066610) (to M.L.D.) and the Department of Defense (W81XWH-08-1-0035) (to C.D.V.). 


\section{REFERENCES}

1. Edwards J, Krishna NS, Grigor KM, Bartlett JM. Androgen receptor gene amplification and protein expression in hormone refractory prostate cancer. Br J Cancer 2003;89(3):552-556.

2. van der Kwast TH, Schalken J, Ruizeveld de Winter JA, van Vroonhoven CC, Mulder E, Boersma W, Trapman J. Androgen receptors in endocrine-therapy-resistant human prostate cancer. Int J Cancer 1991;48(2):189-193.

3. Davis JN, Wojno KJ, Daignault S, Hofer MD, Kuefer R, Rubin MA, Day ML. Elevated E2F1 inhibits transcription of the androgen receptor in metastatic hormone-resistant prostate cancer. Cancer Res 2006;66(24):11897-11906.

4. Engelmann D, Putzer BM. The dark side of E2F1: In transit beyond apoptosis. Cancer Res 2012;72(3):571-575.

5. Rhodes DR, Kalyana-Sundaram S, Mahavisno V, Barrette TR, Ghosh D, Chinnaiyan AM. Mining for regulatory programs in the cancer transcriptome. Nat Genet 2005;37(6):579-583.

6. Malhotra S, Lapointe J, Salari K, Higgins JP, Ferrari M, Montgomery K, van de Rijn M, Brooks JD, Pollack JR. A trimarker proliferation index predicts biochemical recurrence after surgery for prostate cancer. PLoS ONE 2011;6(5):e20293.

7. McCabe MT, Low JA, Daignault S, Imperiale MJ, Wojno KJ, Day ML. Inhibition of DNA methyltransferase activity prevents tumorigenesis in a mouse model of prostate cancer. Cancer Res 2006;66(1):385-392.

8. McCabe DC, Caudill MA. DNA methylation, genomic silencing, and links to nutrition and cancer. Nutr Rev 2005;63(6 Pt 1): 183-195.

9. el-Deiry WS, Nelkin BD, Celano P, Yen RW, Falco JP, Hamilton SR, Baylin SB. High expression of the DNA methyltransferase gene characterizes human neoplastic cells and progression stages of colon cancer. Proc Natl Acad Sci USA 1991;88(8): 3470-3474.

10. Wu CT, Wu CF, Lu CH, Lin CC, Chen WC, Lin PY, Chen MF Expression and function role of DNA methyltransferase 1 in human bladder cancer. Cancer 2011;117(22):5221-5233.

11. Chen MF, Chen WC, Chang YJ, Wu CF, Wu CT. Role of DNA methyltransferase 1 in hormone-resistant prostate cancer. J Mol Med 2010;88(9):953-962.

12. Patra SK, Patra A, Zhao H, Dahiya R. DNA methyltransferase and demethylase in human prostate cancer. Mol Carcinog 2002; 33(3):163-171.

13. Tomlins SA, Mehra R, Rhodes DR, Cao X, Wang L, Dhanasekaran SM, Kalyana-Sundaram S, Wei JT, Rubin MA, Pienta KJ, Shah RB, Chinnaiyan AM. Integrative molecular concept modeling of prostate cancer progression. Nat Genet 2007;39(1):41-51.

14. Kinney SR, Moser MT, Pascual M, Greally JM, Foster BA, Karpf AR. Opposing roles of Dnmt1 in early- and late-stage murine prostate cancer. Mol Cell Biol 2010;30(17):4159-4174.

15. Yu YP, Paranjpe S, Nelson J, Finkelstein S, Ren B, Kokkinakis D, Michalopoulos G, Luo JH. High throughput screening of methylation status of genes in prostate cancer using an oligonucleotide methylation array. Carcinogenesis 2005;26(2):471-479.

16. Valdez CD, Davis JN, Odeh HM, Layfield TL, Cousineau CS, Berton TR, Johnson DG, Wojno KJ, Day ML. Repression of androgen receptor transcription through the E2F1/DNMT1 axis. PLoS ONE 2011;6(9):e25187.

17. Greenberg NM, DeMayo F, Finegold MJ, Medina D, Tilley WD, Aspinall JO, Cunha GR, Donjacour AA, Matusik RJ, Rosen JM.
Prostate cancer in a transgenic mouse. Proc Natl Acad Sci USA 1995;92(8):3439-3443.

18. Zorn CS, Wojno KJ, McCabe MT, Kuefer R, Gschwend JE, Day ML. 5-Aza-2'-deoxycytidine delays androgen-independent disease and improves survival in the transgenic adenocarcinoma of the mouse prostate mouse model of prostate cancer. Clin Cancer Res 2007;13(7):2136-2143.

19. Gingrich JR, Barrios RJ, Kattan MW, Nahm HS, Finegold MJ, Greenberg NM. Androgen-independent prostate cancer progression in the TRAMP model. Cancer Res 1997;57(21):4687-4691.

20. Mutze K, Langer R, Schumacher F, Becker K, Ott K, Novotny A, Hapfelmeier A, Hofler H, Keller G. DNA methyltransferase 1 as a predictive biomarker and potential therapeutic target for chemotherapy in gastric cancer. Eur J Cancer 2011;47(12):18171825 .

21. Jarrard DF, Kinoshita H, Shi Y, Sandefur C, Hoff D, Meisner LF, Chang C, Herman JG, Isaacs WB, Nassif N. Methylation of the androgen receptor promoter $\mathrm{CpG}$ island is associated with loss of androgen receptor expression in prostate cancer cells. Cancer Res 1998;58(23):5310-5314.

22. Takahashi S, Inaguma S, Sakakibara M, Cho YM, Suzuki S, Ikeda Y, Cui L, Shirai T. DNA methylation in the androgen receptor gene promoter region in rat prostate cancers. Prostate 2002; 52(1):82-88.

23. Kinoshita H, Shi Y, Sandefur C, Meisner LF, Chang C, Choon A, Reznikoff CR, Bova GS, Friedl A, Jarrard DF. Methylation of the androgen receptor minimal promoter silences transcription in human prostate cancer. Cancer Res 2000;60(13):3623-3630.

24. Sasaki M, Tanaka Y, Perinchery G, Dharia A, Kotcherguina I, Fujimoto S, Dahiya R. Methylation and inactivation of estrogen, progesterone, and androgen receptors in prostate cancer. J Natl Cancer Inst 2002;94(5):384-390.

25. Nakayama T, Watanabe $M$, Suzuki $H$, Toyota $M$, Sekita $N$, Hirokawa Y, Mizokami A, Ito H, Yatani R, Shiraishi T. Epigenetic regulation of androgen receptor gene expression in human prostate cancers. Lab Invest 2000;80(12):1789-1796.

26. Wilson CM, McPhaul MJ. A and B forms of the androgen receptor are present in human genital skin fibroblasts. Proc Natl Acad Sci USA 1994;91(4):1234-1238.

27. Ahrens-Fath I, Politz O, Geserick C, Haendler B. Androgen receptor function is modulated by the tissue-specific AR45 variant. FEBS J 2005;272(1):74-84.

28. Guo Z, Qiu Y. A new trick of an old molecule: Androgen receptor splice variants taking the stage?. Int J Biol Sci 2011;7(6): 815-822.

29. Hu R, Dunn TA, Wei S, Isharwal S, Veltri RW, Humphreys E, Han M, Partin AW, Vessella RL, Isaacs WB, Bova GS, Luo J. Ligand-independent androgen receptor variants derived from splicing of cryptic exons signify hormone-refractory prostate cancer. Cancer Res 2009;69(1):16-22.

30. Guo Z, Yang X, Sun F, Jiang R, Linn DE, Chen H, Kong X, Melamed J, Tepper CG, Kung HJ, Brodie AM, Edwards J, Qiu Y. A novel androgen receptor splice variant is up-regulated during prostate cancer progression and promotes androgen depletionresistant growth. Cancer Res 2009;69(6):2305-2313.

31. Liegibel UM, Sommer U, Boercsoek I, Hilscher U, Bierhaus A, Schweikert HU, Nawroth P, Kasperk C. Androgen receptor isoforms AR-A and AR-B display functional differences in cultured human bone cells and genital skin fibroblasts. Steroids 2003;68(14):1179-1187. 
32. Robert MF, Morin S, Beaulieu N, Gauthier F, Chute IC, Barsalou A, MacLeod AR. DNMT1 is required to maintain CpG methylation and aberrant gene silencing in human cancer cells. Nat Genet 2003;33(1):61-65.

33. Hoffmann MJ, Engers R, Florl AR, Otte AP, Muller M, Schulz WA. Expression changes in EZH2, but not in BMI-1, SIRT1, DNMT1 or DNMT3B are associated with DNA methylation changes in prostate cancer. Cancer Biol Ther 2007;6(9):14031412 .

34. Issa JP, Garcia-Manero G, Giles FJ, Mannari R, Thomas D, Faderl S, Bayar E, Lyons J, Rosenfeld CS, Cortes J, Kantarjian HM.
Phase 1 study of low-dose prolonged exposure schedules of the hypomethylating agent 5-aza-2'-deoxycytidine (decitabine) in hematopoietic malignancies. Blood 2004;103(5):1635-1640.

35. Cheng JC, Matsen CB, Gonzales FA, Ye W, Greer S, Marquez VE, Jones PA, Selker EU. Inhibition of DNA methylation and reactivation of silenced genes by zebularine. J Natl Cancer Inst 2003;95(5):399-409.

36. Jung Y, Park J, Kim TY, Park JH, Jong HS, Im SA, Robertson KD, Bang YJ. Potential advantages of DNA methyltransferase 1 (DNMT1)-targeted inhibition for cancer therapy. J Mol Med 2007;85(10):1137-1148. 